\title{
Teaching nanosafety
}

\author{
Steffen Foss Hansen and Anders Baun present some of the approaches that they have implemented in \\ their Nanotechnology and the Environment course.
}

$\mathrm{S}$ cience and engineering students are increasingly expected to consider the environmental, health and safety (EHS) aspects of emerging technologies, including nanotechnology. Similarly, the new generation of environmental risk assessors is expected to be able to evaluate the safety of products that entail engineered materials. At the Technical University of Denmark (DTU), we have been teaching a three-week fulltime Nanotechnology and the Environment course for ten years, and we feel that the potential success of such a course depends on presenting the bigger picture as well as taking care of a few specific aspects.

\section{Conveying a burning platform}

To motivate our students, we first provide an introduction to nanotechnology and EHS risks. The most important messages are: uses of nanomaterials are increasing; regulation is scattered at best; and no one knows how to complete meaningful risk assessments of the various applications of nanomaterials. Instead of teaching our students how to do an EHS evaluation/risk assessment of different applications of nanomaterials (for example, carbon nanotubes as conductive materials and antifouling agents on ships' hulls), we challenge them "to boldly go where no man has gone before" and perform two meaningful safety evaluations in interdisciplinary groups of 3-4 students, with us as sparring partners.

\section{Research-based teaching}

Reading is deliberately kept to a minimum and we provide only three scientific reviews to deliver historical context. Instead, we focus on training students to complete their own literature searches and critically evaluate the scientific research that they identify as relevant. To provide the students with some fundamental terminology and clues on how to complete the task, we lecture on aspects known to influence the EHS of nanomaterials: dissolution, aggregation/agglomeration, bioaccumulation and persistency.

\section{Short lectures and long breaks}

The lectures are kept short and focus on introducing a given topic, such as bioaccumulation, and asking how it is measured for industrial chemicals, what is known when it comes to nanomaterials and, finally, what the challenges for interpreting scientific studies are. Numerous three-week graduate courses and $\mathrm{PhD}$ courses are held in June at DTU, turning the campus into a playground for students, and specialists in various fields are often happy to drop into our course and facilitate a discussion on their field of expertise. The value of having frequent breaks cannot be underestimated. Besides giving students time to reflect on their learning, it is also often during the break that we are approached regarding specific issues that the students find hard to understand, have reflected on or have encountered in their group work.

\section{Socratic teaching and dialogue}

The students' attempts to identify information that they can use in their safety evaluation very often lead to more questions than answers. We have therefore structured our course in a manner in which the students can always request additional lectures - for example, if they discover that they need more information on the characterization of the size of nanoparticles. In general, however, we tend to use a Socratic approach to teaching, where we assist the students in asking focused questions, explore their assumptions and stimulate discussion rather than providing answers. In order to provoke critical thinking and stimulate discussion, our lectures are often disrupted by $2-3$ small exercises, such as: discuss A and B with a fellow student for three minutes; calculate $\mathrm{X}, \mathrm{Y}$ and $\mathrm{Z}$; or read this scientific article and extract the key elements of it, which will be subsequently discussed in plenum.

\section{Group work and peer learning}

In order to understand the EHS aspects of nanomaterials, one needs to draw on multiple disciplines. By making students work in interdisciplinary groups, we make use of peer learning and the fact that the students learn a great deal by explaining their knowledge to their peers. In that manner, our environmental engineering students are able to teach our nanotechnology and chemistry students about EHS and make them aware of what the issues might be and what they need to know in order to design hazardous properties or exposure out of their future inventions. On the other hand, our nanotechnology and chemistry students are able to teach our environmental engineering students about what makes nanomaterials fundamentally different from industrial chemicals.

Constructive oral and written feedback A key part of the course is the constructive feedback that we provide to the students. The students have the opportunities to submit drafts of their report and they receive feedback on their introduction three times, their methodology twice and their results and analysis once. Both oral and written feedback is provided within 24 hours so that the students have a chance to integrate it into the next version of their report, and it is always constructive. Having the students submit their work multiple times gives us an opportunity to observe which aspects of our teaching the students might have misinterpreted or which we have presented more effectively.

\section{Teaching-based research}

It was clear in the first years we taught the course that it was unrealistic, due to lack of data, to ask the students to complete a risk assessment similar to the ones we normally do for industrial chemicals. Hence, we decided to turn our course into a laboratory for the exploration of alternative risk assessment frameworks. During the first years, we asked the students to use and evaluate the applicability of the framework developed by Environmental Defense and DuPont. As the field matured, our students have attempted to use the Swiss Precautionary Matrix, chemical safety assessment and, finally, NanoRiskCat and GreenScreenNano. The students' attempts to use various frameworks with our assistance have subsequently supported us in our own research.

Overall, the majority of the students that take our course appreciate our blended learning philosophy. Our next ambition is to turn it into a massive open online course for university students and professionals by mid-2018.

STEFFEN FOSS HANSEN and ANDERS BAUN are in the Department of Environmental Engineering at the Technical University of Denmark, Bygningstorvet Building 115, Kgs. Lyngby, DK-2800, Denmark. e-mail: sfha@env.dtu.dk 